\title{
Soup consumption is associated with a lower dietary energy density and a better diet quality in US adults
}

\author{
Yong $\mathrm{Zhu}^{1}$ and James H. Hollis ${ }^{2 *}$ \\ ${ }^{1}$ Department of Epidemiology, The University of Iowa, Iowa City, IA, USA \\ ${ }^{2}$ Department of Food Science and Human Nutrition, Iowa State University, 220 MacKay Hall, Ames, IA 50011, USA \\ (Submitted 31 July 2013 - Final revision received 10 October 2013 - Accepted 30 October 2013 - First published online 2 January 2014 )
}

\begin{abstract}
Epidemiological studies have revealed that soup consumption is associated with a lower risk of obesity. Moreover, intervention studies have reported that soup consumption aids in body-weight management. However, little is known about mechanisms that can explain these findings. The objective of the present study was to investigate associations between soup consumption and daily energy intake, dietary energy density (ED), nutrient intake and diet quality. Adults aged 19-64 years who participated in the National Health and Nutrition Examination Surveys during 2003-8 were included in the study. Soup consumers were identified from the first dietary recall using the United States Department of Agriculture food codes and combination food type from the dietary data. Compared with non-consumers ( $n$ 9307), soup consumers ( $n$ 1291) had a lower body weight $(P=0 \cdot 002)$, a lower waist circumference $(P=0 \cdot 001)$ and a trend towards a lower total energy intake $(P=0 \cdot 087)$. Soup consumption was associated with a lower dietary ED $(P<0 \cdot 001)$; this was independent of whether data on beverage or water consumption were included. Diet quality, as measured by the Healthy Eating Index 2005, was significantly better in soup consumers $(P=0 \cdot 008)$. Soup consumption was also associated with a reduced intake of total fat and an increased intake of protein, carbohydrate and dietary fibre, as well as several vitamins and minerals $(P<0.05$ for all). However, it was also associated with a higher intake of $\mathrm{Na}(P<0 \cdot 001)$. The relationship between soup consumption and body weight could be due to a reduced dietary ED and an improved diet quality. Consumers need to pay attention to their Na intake and choose low-Na products for a healthier diet.
\end{abstract}

Key words: National Health and Nutrition Examination Survey: Soup: Obesity: Energy density: Diet quality

Obesity is a leading public health problem in the developed countries of the world. In 2009-10, it was estimated that $35.9 \%$ of US adults were obese ${ }^{(1)}$. Identification of factors that reduce the risk of obesity may aid in the development of dietary or behavioural strategies for body-weight management ${ }^{(2,3)}$. Accumulating evidence suggests that soup consumption is associated with a lower body weight or a reduced risk of obesity $^{(4-8)}$. In addition, intervention studies ${ }^{(9,10)}$ have also reported that soup consumption as a component of a weight-loss programme results in a better outcome, compared with a traditional programme. However, it is not clear why soup consumption is beneficial for body-weight management.

Recent laboratory studies ${ }^{(11-13)}$ have shown that soup is a food with a strong satiating property that can suppress shortterm appetite or food intake. This raises the possibility that the beneficial effects of soup consumption on body weight could be due to an effect on energy intake. As these laboratory studies $^{(11-13)}$ are short-term interventions that only followed participants for a few hours after soup consumption, it is possible that the reduced appetite or food intake might have been compensated for by increased food intake at subsequent meals. Studies that investigate the association between soup consumption and daily energy intake in free-living conditions may help answer this question.

It has been shown that reducing the energy density (ED) of foods can reduce energy intake and body weight ${ }^{(14-16)}$. Moreover, epidemiological studies ${ }^{(17,18)}$ have demonstrated a positive relationship between $\mathrm{ED}$ and body weight. In addition, a prospective study has found that participants who increased their dietary ED exhibited a greater weight gain over 8 years than those who decreased their dietary $\mathrm{ED}^{(19)}$. A key characteristic of soup is its high water content, and including it in the diet may lower the ED of the diet. As the human diet consists of various foods and beverages, the association between soup consumption and overall dietary ED warrants further investigation.

Abbreviations: ED, energy density; HEI, Healthy Eating Index; NHANES, National Health and Nutrition Examination Survey; USDA, United States Department of Agriculture.

*Corresponding author: J. H. Hollis, fax +1 515294 8181, email jhollis@iastate.edu 
In addition to an effect on body weight, soup consumption may also improve the diet quality. An epidemiological study $^{(20)}$ of French adults has found that soup consumption is related to a higher dietary intake of vitamins including folate, $\beta$-carotene and vitamin $\mathrm{C}$. To our knowledge, similar studies have not been conducted in the US population. Considering the higher prevalence of obesity in the USA ${ }^{(21)}$ and different dietary habits in different countries, a study using samples representing the general US population would fill the gap.

The objective of the present study was to determine the associations between soup consumption and daily energy intake, dietary ED and nutrient intake using the first dietary recall data collected in the National Health and Nutrition Examination Survey (NHANES). Another objective was to investigate whether soup consumption was associated with Healthy Eating Index (HEI)-2005, a parameter developed by the National Cancer Institute and United States Department of Agriculture (USDA) as a measurement of diet quality ${ }^{(22,23)}$. Based on previous findings, it was hypothesised that soup consumption is associated with a diet that is lower in ED with an increased HEI-2005 score.

\section{Methods}

\section{Data source and study population}

The NHANES ${ }^{(24)}$ is a survey administered by the National Center for Health Statistics to assess the health and nutritional status of non-institutionalised residents of the USA; it consists of an interview with demographic, socio-economic, dietary and health-related questions and physical examinations, as well as laboratory tests for subpopulations. The NHANES dietary interview component was What We Eat in America ${ }^{(25)}$. Since 2001, two $24 \mathrm{~h}$ dietary recalls have been collected. The participants were asked to provide the first $24 \mathrm{~h}$ dietary recall at a mobile examination centre. The second dietary recall was obtained by telephone $3-10 \mathrm{~d}$ later. There were several changes in What We Eat in America across the different survey cycles ${ }^{(25)}$. For example, in the NHANES 2003-4, total choline intake data were not available, and water consumption data were not collected during the $24 \mathrm{~h}$ dietary recall. Since 2005, total choline intake data have been added and water consumption data have been collected during the $24 \mathrm{~h}$ dietary recall. Complete details related to sampling methodology, survey instruments, data processing, quality controls and assurance of NHANES data are available on its website ${ }^{(24)}$. The NHANES were conducted according to the guidelines laid down in the Declaration of Helsinki, and all procedures involving human subjects were approved by the National Center for Health Statistics Research Ethics Review Board. Written consent was obtained from each adult participant and guardian of each child participant.

The NHANES data from the 2003-4, 2005-6 and 2007-8 survey cycles were combined to maximise the statistical power of the present study. These data are publicly available on the NHANES website ${ }^{(24)}$. Participants whose first dietary recall was reliable and met the minimum criteria as determined by the NHANES were initially included ( $n$ 27 181). Among these participants, 16583 participants were further excluded as they met either of the following criteria: pregnant or lactating women $(n$ 797) and age below 19 years or above 64 years ( $n 15$ 868). Children (age $\leq 18$ years) and the elderly (age $\geq 65$ years) were excluded because of their reduced energy intake and different dietary patterns ${ }^{(26,27)}$. The final sample included 10598 eligible participants.

\section{Exposure and outcome variables}

The USDA Food and Nutrient Database for Dietary Studies was used for dietary recall data processing in the NHANES, to assign each individual food to an eight-digit USDA food code, and to calculate portion sizes and nutrient intakes; in addition, a combination food type was assigned by the NHANES if different foods were eaten together (such as cereal with milk) ${ }^{(24)}$. Because of the different sample weight in the two dietary recalls, only data from the first dietary recall were used in the present study.

Soup reported in the first dietary recall was identified by the USDA food code. Detailed information on the USDA food coding scheme is available online ${ }^{(28)}$. The description of each USDA food code was initially screened using the keyword 'soup'. All the identified soup items were manually examined to ensure the accuracy of classification. The final code for soup included all the USDA food codes with the first three digits of $147,283,323,416,584,718,723,735$, 746, 756 and 775, as well as another forty-two unique USDA food codes (e.g. 27114000 for 'beef with soup'). A participant was classified as a soup consumer if he/she reported at least one soup item or had a combination food type of soup in the first $24 \mathrm{~h}$ dietary recall.

Body weight and height were measured by trained NHANES interviewers using standardised procedures with calibrated equipment. BMI was calculated by dividing body weight in $\mathrm{kg}$ by the square of height in $\mathrm{m}\left(\mathrm{kg} / \mathrm{m}^{2}\right)$. Waist circumference was measured by a soft tape placed horizontally just above the iliac crest.

Dietary ED was estimated as the ratio of total energy content:weight of dietary items consumed $(\mathrm{kJ} / \mathrm{g})$. There are several methods to calculate ED depending on whether beverages or water is included in calculations ${ }^{(17,18,29)}$. In the present study, ED was calculated in three ways: (1) ED1, using only foods; (2) ED2, using foods and beverages excluding water; (3) ED3, using foods and beverages including water. USDA food code was used to classify foods, beverages and water. Milk and milk drinks, fruit juice, vegetable juice, alcoholic beverages and non-alcoholic beverages (coffee, tea, soft drinks, etc.) were considered as beverages in the present analysis.

The HEI-2005 was used to assess diet quality ${ }^{(22,23)}$. In the present study, it was calculated based on a sample SAS code provided by the USDA Center for Nutrition Policy and Promotion $^{(30)}$. The sample code used information from the MyPyramid Equivalents Database to convert food intake from the NHANES dietary data to MyPyramid serving sizes 
and computed the total HEI-2005 score and its component scores. The sample code and data files for the MyPyramid Equivalents Database are available online ${ }^{(30)}$.

\section{Covariates}

Demographic variables, including age, sex, race/ethnicity and ratio of family income:poverty, were used as covariates ${ }^{(31,32)}$. In addition, total energy intake from foods and beverages or BMI was adjusted for each specific analysis where appropriate ${ }^{(32,33)}$. For race/ethnicity, the participants were recoded into four ethnic groups: Hispanic; non-Hispanic White; nonHispanic Black; other. The variable 'ratio of family income: poverty' was categorised into three groups: high $(\geq 3.50)$; medium $(1 \cdot 86-3 \cdot 49)$; low $(\leq 1 \cdot 85)^{(34)}$.

\section{Statistical analyses}

Data were analysed using the Statistical Analysis Systems statistical software package version 9.3 (SAS Institute, Inc.). In all the analyses, 6-year sample weight and specific Statistical Analysis Systems survey procedures were used to account for the complex multi-stage design of the NHANES. The $\chi^{2}$ test (Proc SurveyFreq) was applied as a bivariate analysis for a comparison of demographic characteristics between non-consumers and soup consumers. Multivariate regression (Proc SurveyReg) was used to compare the covariate-adjusted BMI and waist circumference between non-consumers and soup consumers. It was also used to assess the associations between soup consumption and total energy intake, dietary ED, daily nutrient intake and HEI-2005 component scores. Observations with missing values were excluded from modelling. Data are presented as weighted percentages with their standard errors or adjusted least-square means with their standard errors where appropriate. Significance was considered as $P<0.05$.

Because water consumption data were not reported during the $24 \mathrm{~h}$ dietary recall in the NHANES 2003-4, a sensitivity analysis for the ED data was carried out using dietary data from the NHANES 2005-8 with a 4-year sample weight.

\section{Results}

\section{Characteristics of the participants}

There were 1291 participants who reported soup consumption in their first dietary recall and 9307 participants who did not report any soup item in their first dietary recall. The weighted percentage was 11.9 (SE 0.5 ) \% for soup consumers and 88.1 (SE 0.5 ) \% for non-consumers, respectively. The demographic characteristics of the participants are summarised in Table 1. Compared with non-consumers, a higher percentage of soup consumers were females $(P=0.023)$ and a lower percentage were non-Hispanic White and non-Hispanic Black $(P<0 \cdot 001)$. However, there was no association between soup consumption and the ratio of family income:poverty $(P=0 \cdot 200)$.

\section{$B M I$ and waist circumference}

Covariate-adjusted BMI and waist circumference were significantly lower in soup consumers $(P=0.002$ and 0.001, respectively). Adjusting for age, sex, race, ratio of family income:poverty and energy intake, the least-square mean of BMI was found to be 27.7 ( $\mathrm{SE} 0.3) \mathrm{kg} / \mathrm{m}^{2}$ in soup consumers. It was $28.7(\operatorname{se~} 0 \cdot 1) \mathrm{kg} / \mathrm{m}^{2}$ in non-consumers. Soup consumers had a mean waist circumference of $95.0(\mathrm{SE} 0.7) \mathrm{cm}$; the waist circumference was $97.5(\mathrm{SE} 0.3) \mathrm{cm}$ in non-consumers.

\section{Energy intake and dietary energy density}

The least-square mean of total energy intake, adjusting for demographic covariates and BMI, was 9311 (SE 164) kJ in soup consumers. It was 9599 (SE 76) kJ in non-consumers with a trend towards a significant difference between the two groups $(P=0 \cdot 087)$. Table 2 summarises the covariate-adjusted dietary ED for soup consumers and non-consumers. Soup consumption was found to be associated with a lower

Table 1. Characteristics of participants aged 19-64 years by soup consumption status: National Health and Nutrition Examination Survey 2003-8

(Weighted percentages with their standard errors)

\begin{tabular}{|c|c|c|c|c|c|c|c|}
\hline & \multicolumn{3}{|c|}{ Soup consumers ( $n$ 1291) } & \multicolumn{3}{|c|}{ Non-consumers (n 9307) } & \multirow[b]{2}{*}{$P^{*}$} \\
\hline & $n$ & Weighted percentage & SE & $n$ & Weighted percentage & $\mathrm{SE}$ & \\
\hline Sex & & & & & & & 0.023 \\
\hline Male & 633 & $47 \cdot 3$ & 1.5 & 4787 & $51 \cdot 0$ & 0.5 & \\
\hline Female & 658 & $52 \cdot 7$ & 1.5 & 4520 & $49 \cdot 0$ & 0.5 & \\
\hline Race & & & & & & & $<0.001$ \\
\hline Hispanic & 526 & $19 \cdot 1$ & 1.7 & 2378 & $12 \cdot 2$ & $1 \cdot 1$ & \\
\hline Non-Hispanic White & 501 & 63.4 & 2.5 & 4204 & $70 \cdot 2$ & $2 \cdot 0$ & \\
\hline Non-Hispanic Black & 159 & $6 \cdot 3$ & 0.7 & 2361 & $12 \cdot 6$ & $1 \cdot 2$ & \\
\hline Other & 105 & $11 \cdot 2$ & 1.7 & 364 & $5 \cdot 0$ & 0.4 & \\
\hline Ratio of family income:poverty & & & & & & & $0 \cdot 200$ \\
\hline Less or equal to 1.85 & 527 & $29 \cdot 4$ & 1.8 & 3620 & $28 \cdot 5$ & $1 \cdot 2$ & \\
\hline Between 1.85 and 3.50 & 254 & $22 \cdot 0$ & $1 \cdot 6$ & 2121 & $25 \cdot 1$ & $1 \cdot 0$ & \\
\hline Greater or equal to 3.50 & 417 & $48 \cdot 6$ & $2 \cdot 4$ & 3008 & $46 \cdot 4$ & 1.6 & \\
\hline
\end{tabular}

${ }^{*} \chi^{2} P$ value was obtained from the bivariate analysis for the comparison between two groups.

†Total $n$ was less than 10598 due to missing values. 
Table 2. Dietary energy density (ED) of participants aged 19-64 years by soup consumption status: National Health and Nutrition Examination Survey 2003-8

(Least-square mean (LSM) values with their standard errors)

\begin{tabular}{|c|c|c|c|c|c|}
\hline & \multicolumn{2}{|c|}{$\begin{array}{l}\text { Soup consumers } \\
\quad(n 1291)\end{array}$} & \multicolumn{2}{|c|}{$\begin{array}{l}\text { Non-consumers } \\
\quad(n \text { 9307) }\end{array}$} & \multirow[b]{2}{*}{$P \dagger$} \\
\hline & LSM & $S E^{*}$ & LSM & $\mathrm{SE}^{*}$ & \\
\hline ED1 (kJ/g)‡ & 6.41 & $0 \cdot 10$ & 8.81 & 0.06 & $<0.001$ \\
\hline ED2 $(\mathrm{kJ} / \mathrm{g}) \S$ & 3.58 & 0.06 & 4.04 & 0.03 & $<0.001$ \\
\hline ED3 (kJ/g)\| & $2 \cdot 34$ & 0.04 & $2 \cdot 65$ & 0.03 & $<0.001$ \\
\hline
\end{tabular}

* Data were adjusted for age, sex, race, ratio of family income:poverty and BMI. $\dagger P$ value for the comparison between soup consumers and non-consumers. $\ddagger E D$ estimated as energy from all food items divided by weight of all food items. $\S E D$ estimated as energy from all foods and beverages excluding water divided by weight of all foods and beverages excluding water.

|| ED estimated as energy from all foods and beverages including water divided by weight of all foods and beverages including water.

dietary ED, which was independent of the method used to calculate ED $(P<0.001$ for all). The sensitivity analysis using dietary data from the NHANES 2005-8 yielded a similar result for dietary ED (data not shown).

\section{Nutrient intake}

Table 3 summarises the covariate-adjusted nutrient intake by the status of soup consumption. Soup consumption was found to be associated with a reduced intake of total fat $(P<0.001)$ and an increased intake of protein $(P=0.036)$, carbohydrate $(P=0.003)$, dietary fibre $(P<0.001)$, vitamin A $(P<0.001)$ and vitamin $C(P=0.002)$. In addition, the intake of minerals such as $\mathrm{P}, \mathrm{Mg}, \mathrm{Fe}, \mathrm{Cu}$ and $\mathrm{K}$ was higher $(P<0 \cdot 01$ for all). Nonetheless, the intake of $\mathrm{Na}$ was also higher in soup consumers $(P<0 \cdot 001)$.

\section{Diet quality}

The total HEI-2005 score and each component score are summarised in Table 4. Soup consumers had a better diet quality, revealed by a higher total HEI-2005 score $(P=0 \cdot 008)$. Compared with non-consumers, soup consumers had significantly higher intakes of most MyPyramid food groups including total fruits $(P<0.001)$, whole fruits $(P=0.004)$, total vegetables $(P<0.001)$, dark green/orange vegetables/legumes $(P<0.001)$ and total grains $(P=0.005)$. A lower score was observed for oil $(P=0 \cdot 027)$. In addition, soup consumers had a better score for saturated fat $(P<0.001)$, as well as solid fats/alcohol/added sugars $(P=0 \cdot 002)$. In agreement with the nutrient data indicating that soup consumption is associated with a higher intake of $\mathrm{Na}$, a lower score was observed for $\mathrm{Na}$ in soup consumers $(P<0 \cdot 001)$.

\section{Discussion}

In agreement with previous studies ${ }^{(4-8)}$ that have investigated the associations between soup consumption and body weight, the results of the present study using nationally representative data demonstrated that soup consumption was inversely related to body weight and waist circumference. Daily energy intake tended to be lower in soup consumers. In addition, soup consumption was associated with a lower dietary ED and a higher total HEI-2005 score.

The satiating properties of soup have been studied by Mattes ${ }^{(11)}$, who compared the difference in appetite and energy intake in response to an isoenergetic preload of apple juice, apple soup or apple. Apple soup was found to result in a lower rating for postprandial hunger and the median $24 \mathrm{~h}$ energy intake was the lowest when apple soup was consumed ${ }^{(11)}$. Flood \& Rolls ${ }^{(12)}$ found that total energy intake from a soup preload and an ad libitum meal served 15 min later was significantly lower than energy intake from an ad libitum meal without a soup preload. A recent study carried out by Clegg et al. ${ }^{(13)}$ has revealed that the appetite-suppressing effect of soup is due to a delayed gastric-emptying rate and an increased glycaemic response. By contrast, results obtained for energy intake in the present study revealed only a marginally significant difference. The smaller percentage of identified soup consumers (11.9\%) in the present study may have resulted in an insufficient statistical power for the comparison between soup consumers and non-consumers. The difference between the results of the present study and those of previous laboratory studies $^{(11,12)}$ could possibly be due to dietary habits or the

Table 3. Nutrient intake of participants aged 19-64 years by soup consumption status: National Health and Nutrition Examination Survey 2003-8

(Least-square means (LSM) values with their standard errors)

\begin{tabular}{|c|c|c|c|c|c|}
\hline \multirow[b]{2}{*}{ Nutrient intake* } & \multicolumn{2}{|c|}{$\begin{array}{l}\text { Soup consumers } \\
\quad(n 1291)\end{array}$} & \multicolumn{2}{|c|}{$\begin{array}{l}\text { Non-consumers } \\
\quad(n \text { 9307) }\end{array}$} & \multirow[b]{2}{*}{$P \dagger$} \\
\hline & LSM & SE & LSM & SE & \\
\hline Protein $(\mathrm{g})$ & $88 \cdot 1$ & 1.0 & $86 \cdot 0$ & 0.5 & 0.036 \\
\hline Total fat $(\mathrm{g})$ & $81 \cdot 8$ & 0.7 & $86 \cdot 4$ & 0.4 & $<0.001$ \\
\hline SFA (g) & $27 \cdot 2$ & 0.4 & $28 \cdot 7$ & $0 \cdot 1$ & 0.001 \\
\hline MUFA (g) & $30 \cdot 3$ & 0.3 & $32 \cdot 1$ & 0.2 & $<0.001$ \\
\hline PUFA (g) & $17 \cdot 2$ & 0.3 & $18 \cdot 1$ & 0.2 & 0.007 \\
\hline Cholesterol (mg) & $291 \cdot 3$ & $8 \cdot 3$ & 303.7 & $3 \cdot 1$ & 0.152 \\
\hline Carbohydrate (g) & $280 \cdot 7$ & $2 \cdot 4$ & $273 \cdot 1$ & 1.4 & 0.003 \\
\hline Total dietary fibre $(\mathrm{g})$ & $17 \cdot 6$ & 0.4 & $15 \cdot 4$ & 0.2 & $<0.001$ \\
\hline Alcohol (g) & $12 \cdot 3$ & $1 \cdot 1$ & 11.9 & 0.4 & 0.749 \\
\hline Vitamin $A(\mu \mathrm{g}) \ddagger$ & 665.7 & $16 \cdot 2$ & 578.0 & $10 \cdot 9$ & $<0.001$ \\
\hline Vitamin E (mg)§ & $7 \cdot 6$ & 0.2 & 7.4 & 0.1 & 0.190 \\
\hline Vitamin C (mg) & $97 \cdot 1$ & 3.9 & $84 \cdot 1$ & 2.5 & 0.002 \\
\hline Vitamin $\mathrm{K}(\mu \mathrm{g})$ & $101 \cdot 4$ & $5 \cdot 0$ & 93.3 & 2.5 & $0 \cdot 160$ \\
\hline Thiamin (mg) & 1.74 & 0.05 & 1.68 & 0.01 & 0.283 \\
\hline Riboflavin (mg) & 2.35 & 0.05 & $2 \cdot 26$ & 0.02 & 0.059 \\
\hline Niacin (mg) & $26 \cdot 5$ & 0.4 & $25 \cdot 7$ & 0.2 & 0.062 \\
\hline Vitamin $B_{6}(\mathrm{mg})$ & $2 \cdot 04$ & 0.04 & 1.96 & 0.02 & $0 \cdot 121$ \\
\hline Total folate $(\mu \mathrm{g})$ & $423 \cdot 4$ & $8 \cdot 2$ & $406 \cdot 4$ & 4.5 & 0.074 \\
\hline Vitamin $B_{12}(\mu \mathrm{g})$ & $5 \cdot 6$ & $0 \cdot 2$ & $5 \cdot 3$ & $0 \cdot 1$ & 0.258 \\
\hline $\mathrm{Ca}(\mathrm{mg})$ & 957.2 & $19 \cdot 7$ & $938 \cdot 2$ & $9 \cdot 7$ & 0.346 \\
\hline$P(\mathrm{mg})$ & $1424 \cdot 0$ & 14.5 & 1372.5 & $7 \cdot 2$ & 0.001 \\
\hline $\mathrm{Mg}(\mathrm{mg})$ & $318 \cdot 1$ & $4 \cdot 2$ & 292.6 & $2 \cdot 7$ & $<0.001$ \\
\hline $\mathrm{Fe}(\mathrm{mg})$ & $16 \cdot 6$ & 0.2 & $15 \cdot 7$ & $0 \cdot 1$ & $<0.001$ \\
\hline $\mathrm{Zn}(\mathrm{mg})$ & $12 \cdot 6$ & $0 \cdot 2$ & $12 \cdot 6$ & 0.1 & 0.843 \\
\hline $\mathrm{Cu}(\mu \mathrm{g})$ & 1.47 & 0.02 & 1.34 & 0.02 & $<0.001$ \\
\hline $\mathrm{Na}(\mathrm{mg})$ & $4135 \cdot 3$ & $80 \cdot 6$ & $3537 \cdot 2$ & $17 \cdot 4$ & $<0.001$ \\
\hline $\mathrm{K}(\mathrm{mg})$ & 3004.2 & $42 \cdot 8$ & $2672 \cdot 6$ & 17.4 & $<0.001$ \\
\hline
\end{tabular}

*Data were adjusted for age, sex, race, ratio of family income:poverty and energy intake.

$\dagger P$ value for the comparison between soup consumers and non-consumers. $\ddagger$ Vitamin $A$ as retinol activity equivalents.

$\S$ Vitamin $\mathrm{E}$ as $\alpha$-tocopherol. 
Table 4. Healthy Eating Index (HEI)-2005 total score and component score of participants aged 19-64 years by soup consumption status: National Health and Nutrition Examination Survey 2003-8 (Least-square mean (LSM) values with their standard errors)

\begin{tabular}{|c|c|c|c|c|c|}
\hline \multirow[b]{2}{*}{ HEI-2005 component score (maximum)* } & \multicolumn{2}{|c|}{$\begin{array}{l}\text { Soup consumers } \\
(n 1291)\end{array}$} & \multicolumn{2}{|c|}{$\begin{array}{l}\text { Non-consumers } \\
\quad(n \text { 9307) }\end{array}$} & \multirow[b]{2}{*}{$P \dagger$} \\
\hline & LSM & SE & LSM & SE & \\
\hline Total HEI-2005 (100) & $51 \cdot 10$ & 0.63 & $49 \cdot 18$ & 0.39 & 0.008 \\
\hline Total fruits $(5)$ & $2 \cdot 22$ & 0.08 & 1.87 & 0.06 & $<0.001$ \\
\hline Whole fruits (5) & 1.98 & 0.08 & 1.68 & 0.05 & 0.004 \\
\hline Total vegetables (5) & 3.32 & 0.07 & $2 \cdot 82$ & 0.03 & $<0.001$ \\
\hline Dark green/orange vegetables/legumes (5) & 1.58 & 0.06 & 1.00 & 0.03 & $<0.001$ \\
\hline Total grains (5) & $4 \cdot 19$ & 0.05 & 4.05 & 0.02 & 0.005 \\
\hline Whole grains (5) & 0.91 & 0.05 & 0.89 & 0.03 & 0.679 \\
\hline Milk (10) & $4 \cdot 70$ & 0.19 & 4.61 & 0.07 & 0.615 \\
\hline Meat and beans (10) & 8.02 & 0.13 & $8 \cdot 16$ & 0.05 & 0.318 \\
\hline Oils (10) & $5 \cdot 12$ & 0.16 & 5.52 & 0.07 & 0.027 \\
\hline Saturated fat (10) & $6 \cdot 30$ & 0.17 & 5.62 & 0.06 & $<0.001$ \\
\hline $\mathrm{Na}(10)$ & $2 \cdot 68$ & 0.16 & $4 \cdot 17$ & 0.05 & $<0.001$ \\
\hline SoFAAS energy (20) & 10.08 & 0.35 & $8 \cdot 80$ & 0.18 & 0.002 \\
\hline
\end{tabular}

SoFAAS, solid fats/alcohol/added sugars.

* Data were adjusted for age, sex, race, ratio of family income:poverty and BMI.

$\dagger P$ value for the comparison between soup consumers and non-consumers.

possibility that food choices may differ between laboratory and free-living conditions. Indeed, the study carried out by Mattes ${ }^{(11)}$ revealed that apple soup received the lowest hedonic ratings among the test foods. Under free-living conditions, people may choose other foods if available, instead of eating a food that is less pleasant or palatable. Another possibility is that if soup was consumed as the last meal on the dietary recall day, the effect on postprandial appetite, if any, would not play an important role in the contribution to total energy intake as no further food is consumed subsequently on that day. In addition, some studies have shown that reduced appetite during a postprandial period may be short-lived and does not result in changed food intake at subsequent meals ${ }^{(35,36)}$. It remains unknown how long the appetite-suppressing effect of soup can last.

Previous studies using NHANES data had shown that dietary ED was positively related to body weight in both children ${ }^{(17)}$ and adults ${ }^{(18)}$, although such associations disappeared when beverages were included in the calculation of $\operatorname{ED}^{(17,18)}$. Because of the positive relationship between ED and body weight, BMI was included as another covariate in the analysis of ED data. The results indicated that when soup was consumed, covariate-adjusted dietary ED was significantly lower, even if water or beverages were included in the calculation of ED. These data suggest that a lower dietary ED could be a possible explanation for the beneficial effect of soup on body weight observed in weight-loss programmes ${ }^{(9,10)}$.

In the present study, soup consumption was found to be associated with a better diet quality as revealed by a higher total score of HEI-2005. A number of dietary factors, such as fruit or fruit juice consumption, are associated with a better diet quality ${ }^{(37,38)}$. The results of the present study indicated that the HEI-2005 component scores for both total fruits and whole fruits were higher when soup was included in the diet. In addition, scores for total vegetables as well as dark green or orange vegetables and legumes were also higher in soup consumers. The inclusion of fruits or vegetables in the diet may further reduce ED due to their higher water content. A higher intake of fruits or vegetables is associated with a reduced risk of weight gain ${ }^{(39,40)}$, which may partly explain the beneficial effect of soup consumption on body weight. Another reason could be that soup consumption is related to a better score for solid fats/alcohol/added sugars, suggesting that energy from solid fats, alcohol and added sugars in the diet is lower in soup consumers. Moreover, the lower scores for oil in soup consumers, together with nutrient data indicating reduced fat intake when soup is consumed, indicate that low energy intake from dietary fat may have contributed to a lower body weight observed in this population.

In the present study, it was found that soup consumption was related to increased dietary fibre intake. Dietary fibre exerts beneficial effects on appetite ${ }^{(41)}$, glycaemic control ${ }^{(42)}$ and gastrointestinal heath ${ }^{(43)}$ due to its rheological properties and its fermentability ${ }^{(44)}$. The effect of dietary fibre on body weight was reviewed by Pereira \& Ludwig ${ }^{(45)}$. It was suggested that dietary fibre may aid in body-weight regulation as reduction in insulin secretion or energy intake when dietary fibre was ingested would contribute to increased fat oxidation and reduced fat storage ${ }^{(45)}$. Therefore, increased dietary fibre intake associated with soup consumption may provide an additional explanation for those studies that have shown that soup consumption has beneficial effects on body weight ${ }^{(9,10)}$.

In the present study, it was also found that soup consumption was associated with a higher intake of vitamins $\mathrm{A}$ and $\mathrm{C}$, as well as several minerals. Similar results have been reported from a cross-sectional survey in a French population ${ }^{(20)}$. Moreover, randomised controlled trials have also shown that soup consumption over several weeks results in a better bioavailability of carotenoids ${ }^{(46)}$ or vitamin $\mathrm{C}^{(47)}$. Although the results of the present study support these findings, it was observed that soup consumption was also 
related to a higher intake of $\mathrm{Na}$, with a corresponding result of a lower score for the Na component of HEI-2005. At this stage, it remains unclear whether the excessive $\mathrm{Na}$ intake was from soup itself or other dietary sources. Considering the negative impact of high $\mathrm{Na}$ intake on cardiovascular health ${ }^{(48)}$ and mortality $^{(49)}$, it is recommended that low-Na soup products be chosen and the amount of table salt used in food processing and preparation be reduced.

The study has some limitations. First, although the NHANES used a number of approaches to help participants provide a more accurate dietary recall, such as provision of tools for the estimation of food portion size, it may still be subject to a self-report bias. Second, although intervention studies have shown that soup consumption has a beneficial effect on body weight ${ }^{(9,10)}$, the association between soup consumption and body weight observed in the present study does not indicate any causal relationship as it was a cross-sectional survey. Third, soup consumers were identified using a $24 \mathrm{~h}$ dietary recall; therefore, the non-consumer group in the present study may consist of people who consumed soup regularly but did not consume soup on the dietary recall day. In addition, it was observed that the participants frequently consumed soup with other foods at the same time; as a result, the type of soup consumed was not investigated due to the complexity of soup components. Future studies are required to determine whether the type of soup consumed has different effects. Despite these limitations, the present study is the first to show that when soup is included in the diet in free-living conditions, it is associated with a better diet quality and a reduced dietary ED. This may provide mechanistic explanations for intervention studies showing a beneficial effect of soup consumption on body weight. Nonetheless, as high $\mathrm{Na}$ intake is also associated with soup consumption, consumers should be aware of this and choose products low in $\mathrm{Na}$ for a healthier diet.

\section{Acknowledgements}

The authors thank the participants and researchers of the NHANES who made the data available. The present study received no specific grant from any funding agency or commercial or not-for-profit sectors. Y. Z. and J. H. H. designed the present study. Y. Z. carried out the data analysis. Y. Z. and J. H. H. prepared the manuscript. The authors declare no conflicts of interest.

\section{References}

1. Flegal KM, Carroll MD, Kit BK, et al. (2012) Prevalence of obesity and trends in the distribution of body mass index among US adults, 1999-2010. J Am Med Assoc 307, 491- 497 .

2. Swinburn BA, Caterson I, Seidell JC, et al. (2004) Diet, nutrition and the prevention of excess weight gain and obesity. Public Health Nutr 7, 123-146.

3. Zhu Y \& Hollis JH (2013) Appetite control and regulation of food intake: a review of proposed mechanism. Nutr Ther Metab 31, 58-68.
4. Bessa M, Valente H, Cordeiro T, et al. (2008) Fluid intake and overweight risk in children (in Portuguese). Acta Medica Port 21, 161-169.

5. Giacosa A \& Filiberti R (1997) Determination of behavior of food consumption in the local area (in Italian). Med Doctor $\mathbf{8}, 40-45$.

6. Kuroda M, Ohta M, Okufuji T, et al. (2011) Frequency of soup intake is inversely associated with body mass index, waist circumference, and waist-to-hip ratio, but not with other metabolic risk factors in Japanese men. J Am Diet Assoc 111, 137-142.

7. Bertrais S, Galan P, Renault N, et al. (2001) Consumption of soup and nutritional intake in French adults: consequences for nutritional status. J Hum Nutr Diet 14, 121-128.

8. Moreira P \& Padrao P (2006) Educational, economic and dietary determinants of obesity in Portuguese adults: a cross-sectional study. Eat Behav 7, 220-228.

9. Jordan HA, Levitz LS, Utgoff KL, et al. (1981) Role of food characteristics in behavioral change and weight loss. $J \mathrm{Am}$ Diet Assoc 79, 24-29.

10. Foreyt JP, Reeves RS, Darnell LS, et al. (1986) Soup consumption as a behavioral weight loss strategy. J Am Diet Assoc $\mathbf{8 6}$, $524-526$.

11. Mattes R (2005) Soup and satiety. Physiol Behav 83, 739-747.

12. Flood JE \& Rolls BJ (2007) Soup preloads in a variety of forms reduce meal energy intake. Appetite 49, 626-634.

13. Clegg ME, Ranawana V, Shafat A, et al. (2013) Soups increase satiety through delayed gastric emptying yet increased glycaemic response. Eur J Clin Nutr 67, 8-11.

14. Bell EA, Castellanos VH, Pelkman CL, et al. (1998) Energy density of foods affects energy intake in normal-weight women. Am J Clin Nutr 67, 412-420.

15. Latner JD, Rosewall JK \& Chisholm AM (2008) Energy density effects on food intake, appetite ratings, and loss of control in women with binge eating disorder and weightmatched controls. Eat Behav 9, 257-266.

16. Ello-Martin JA, Ledikwe JH \& Rolls BJ (2005) The influence of food portion size and energy density on energy intake: implications for weight management. Am J Clin Nutr $\mathbf{8 2}$, 236S-241S.

17. Vernarelli JA, Mitchell DC, Hartman TJ, et al. (2011) Dietary energy density is associated with body weight status and vegetable intake in US children. J Nutr 141, 2204-2210.

18. Kant AK \& Graubard BI (2005) Energy density of diets reported by American adults: association with food group intake, nutrient intake, and body weight. Int $J$ Obes $\mathbf{2 9}$, 950-956.

19. Bes-Rastrollo M, van Dam RM, Martinez-Gonzalez MA, et al (2008) Prospective study of dietary energy density and weight gain in women. Am J Clin Nutr 88, 769-777.

20. Galan P, Renault N, Aissa M, et al. (2003) Relationship between soup consumption, folate, beta-carotene, and vitamin $\mathrm{C}$ status in a French adult population. Int J Vitam Nutr Res 73, 315-321.

21. Low S, Chin MC \& Deurenberg-Yap M (2009) Review on epidemic of obesity. Ann Acad Med Singapore 38, 57-65.

22. Guenther PM, Reedy J \& Krebs-Smith SM (2008) Development of the Healthy Eating Index-2005. J Am Diet Assoc 108, 1896-1901.

23. Guenther PM, Reedy J, Krebs-Smith SM, et al. (2008) Evaluation of the Healthy Eating Index-2005. J Am Diet Assoc 108 $1854-1864$.

24. Centers for Disease Control and Prevention (2013) National Health and Nutrition Examination Survey. http://www.cdc. gov/nchs/nhanes.htm (accessed January 2013). 
25. Centers for Disease Control and Prevention (2010) What we eat in America. http://www.cdc.gov/nchs/nhanes/wweia.htm (accessed September 2013)

26. Wurtman JJ, Lieberman H, Tsay R, et al. (1988) Calorie and nutrient intakes of elderly and young subjects measured under identical conditions. J Gerontol 43, B174-B180.

27. Briefel RR \& Johnson CL (2004) Secular trends in dietary intake in the United States. Annu Rev Nutr 24, 401-431.

28. United States Department of Agriculture (2013) Food Coding Scheme. http://www.ars.usda.gov/Services/docs.htm?docid $=12074$ (accessed January 2013)

29. Vernarelli JA, Mitchell DC, Rolls BJ, et al. (2013) Methods for calculating dietary energy density in a nationally representative sample. Procedia Food Sci 2, 68-74.

30. US Department of Agriculture (2007) Healthy Eating Index-2005 development and evaluation technical report support files. http://www.cnpp.usda.gov/HealthyEatingIndex-2005report. htm (accessed January 2013).

31. Wang Y \& Beydoun MA (2009) Meat consumption is associated with obesity and central obesity among US adults. Int J Obes 33, 621-628.

32. Papanikolaou Y \& Fulgoni VL (2008) Bean consumption is associated with greater nutrient intake, reduced systolic blood pressure, lower body weight, and a smaller waist circumference in adults: results from the National Health and Nutrition Examination Survey 1999-2002. J Am Coll Nutr 27, 569-576.

33. O'Neil CE, Keast DR, Nicklas TA, et al. (2011) Nut consumption is associated with decreased health risk factors for cardiovascular disease and metabolic syndrome in U.S. adults: NHANES 1999-2004. J Am Coll Nutr 30, 502-510.

34. Brauchla M, Juan W, Story J, et al. (2012) Sources of dietary fiber and the association of fiber intake with childhood obesity risk (in 2-18 year olds) and diabetes risk of adolescents $12-18$ year olds: NHANES 2003-2006. J Nutr Metab 2012, 736258.

35. Zhu Y, Hsu WH \& Hollis JH (2013) Increasing the number of masticatory cycles is associated with reduced appetite and altered postprandial plasma concentrations of gut hormones, insulin and glucose. Br J Nutr 110, 384-390.

36. Zhu Y, Hsu WH \& Hollis JH (2013) The effect of food form on satiety. Int J Food Sci Nutr 64, 385-391.

37. O'Neil CE, Nicklas TA, Zanovec M, et al. (2011) Diet quality is positively associated with $100 \%$ fruit juice consumption in children and adults in the United States: NHANES 2003-2006. Nutr J 10, 17.
38. Keast DR, O'Neil CE \& Jones JM (2011) Dried fruit consumption is associated with improved diet quality and reduced obesity in US adults: National Health and Nutrition Examination Survey, 1999-2004. Nutr Res 31, 460-467.

39. Vioque J, Weinbrenner T, Castello A, et al. (2008) Intake of fruits and vegetables in relation to 10 -year weight gain among Spanish adults. Obesity 16, 664-670.

40. Buijsse B, Feskens EJM, Schulze MB, et al. (2009) Fruit and vegetable intakes and subsequent changes in body weight in European populations: results from the project on Diet, Obesity, and Genes (DiOGenes). Am J Clin Nutr 90, 202-209.

41. Zhu Y \& Hollis JH (2013) The impact of food viscosity on eating rate, subjective appetite, glycemic response and gastric emptying rate. PLoS One 8, e67482.

42. Panahi S, Ezatagha A, Temelli F, et al. (2007) Beta-glucan from two sources of oat concentrates affect postprandial glycemia in relation to the level of viscosity. J Am Coll Nutr 26, 639-644.

43. Tantamango YM, Knutsen SF, Beeson L, et al. (2011) Association between dietary fiber and incident cases of colon polyps: the adventist health study. Gastrointest Cancer Res 4, 161-167.

44. Anderson JW, Baird P, Davis RH, et al. (2009) Health benefits of dietary fiber. Nutr Rev 67, 188-205.

45. Pereira MA \& Ludwig DS (2001) Dietary fiber and bodyweight weight regulation: observations and mechanisms. Pediatr Clin North Am 48, 969-980.

46. Martinez-Tomas $\mathrm{R}$, Larque $\mathrm{E}$, Gonzalez-Silvera $\mathrm{D}$, et al. (2012) Effect of the consumption of a fruit and vegetable soup with high in vitro carotenoid bioaccessibility on serum carotenoid concentrations and markers of oxidative stress in young men. Eur J Nutr 51, 231-239.

47. Sanchez-Moreno C, Cano MP, de Ancos B, et al. (2006) Mediterranean vegetable soup consumption increases plasma vitamin $\mathrm{C}$ and decreases $\mathrm{F}$-2-isoprostanes, prostaglandin E-2 and monocyte chemotactic protein-1 in healthy humans. J Nutr Biochem 17, 183-189.

48. He JA, Ogden LG, Vupputuri S, et al. (1999) Dietary sodium intake and subsequent risk of cardiovascular disease in overweight adults. J Am Med Assoc 282, 2027-2034.

49. Alderman MH, Cohen H \& Madhavan S (1998) Dietary sodium intake and mortality: the National Health and Nutrition Examination Survey (NHANES I). Lancet 351, 781-785. 